\section{BRAZIULIAN JOURNAL}

OF MEDICAL AND BIOLOGICAL RLSH.ARCH

www.bjournal.com.br
ISSN 0100-879X

Volume 43 (01) 1-123 January 2010

BIOMEDICAL SCIENCES

AND

CLINICAL INVESTIGATION

Braz J Med Biol Res, J anuary 2010, Volume 43(1) 8-12

Characterization of the interdependency between residues that bind the substrate in a $\beta$-glycosidase

M.H. Tomassi, J.H.K. Rozenfeld, L.M. Gonçalves and S.R. Marana

The Brazilian Journal of Medical and Biological Research is partially financed by
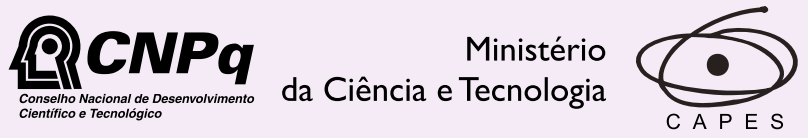

Ministério da Educação

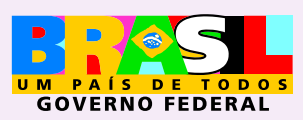

Institutional Sponsors 


\title{
Characterization of the interdependency between residues that bind the substrate in a $\beta$-glycosidase
}

\author{
M.H. Tomassi*, J.H.K. Rozenfeld*, L.M. Gonçalves and S.R. Marana \\ Departamento de Bioquímica, Instituto de Química, Universidade de São Paulo, São Paulo, SP, Brasil
}

\begin{abstract}
The manner by which effects of simultaneous mutations combine to change enzymatic activity is not easily predictable because these effects are not always additive in a linear manner. Hence, the characterization of the effects of simultaneous mutations of amino acid residues that bind the substrate can make a significant contribution to the understanding of the substrate specificity of enzymes. In the $\beta$-glycosidase from Spodoptera frugiperda ( $\mathrm{Sf} \beta \mathrm{gly}$ ), both residues Q39 and E451 interact with the substrate and this is essential for defining substrate specificity. Double mutants of Sfßgly (A451E39, S451E39 and S451N39) were prepared by site-directed mutagenesis, expressed in bacteria and purified using affinity chromatography. These enzymes were characterized using $p$-nitrophenyl $\beta$-galactoside and $p$-nitrophenyl $\beta$-fucoside as substrates. The $k_{\text {cat }} / K_{m}$ ratio for single and double mutants of Sfßgly containing site-directed mutations at positions Q39 and E451 was used to demonstrate that the effect on the free energy of ES‡ (enzyme-transition state complex) of the double mutations $\left(\Delta \Delta G^{\ddagger} x y\right)$ is not the sum of the effects resulting from the single mutations $\left(\Delta \Delta G^{\ddagger}\right.$ and $\Delta \Delta G_{y}^{\ddagger}$ ). This difference in $\Delta \Delta G^{\ddagger}$ indicates that the effects of the single mutations partially overlap. Hence, this common effect counts only once in $\Delta \Delta G{ }_{x y}$. Crystallographic data on $\beta$-glycosidases reveal the presence of a bidentate hydrogen bond involving residues Q39 and E451 and the same hydroxyl group of the substrate. Therefore, both thermodynamic and crystallographic data suggest that residues Q39 and E451 exert a mutual influence on their respective interactions with the substrate.
\end{abstract}

Key words: $\beta$-glycosidase; Substrate specificity; Site-directed mutagenesis; Spodoptera frugiperda

\section{Introduction}

The rational design of enzymes usually requires information from two or more simultaneous mutations. However, the manner in which effects of simultaneous mutations combine to change enzymatic activity is not easily predictable because these effects on enzyme activity are not always additive in a linear manner (1-4).

The interaction between the residues that bind the substrate can be detected by comparing the effect of double mutations $\left(\Delta \Delta G^{\ddagger} x y\right)$ on the free energy of the ES (enzyme-transition state complex) with that of the corresponding single mutations $\left(\Delta \Delta G^{\ddagger} x\right.$ and $\left.\Delta \Delta G^{\ddagger} y\right)$. The difference between $\Delta \Delta G^{\ddagger}{ }_{x y}$ and the sum of $\Delta \Delta G^{\ddagger}{ }_{x}$ and $\Delta \Delta G^{\ddagger}$, known as "coupling energy" ( $\Delta G^{\ddagger}$ I), corresponds to the extent to which the interaction between two residues ( $x$ and $y$ ) affects the stabilization of ES $\ddagger$ and consequently the substrate specificity of enzymes (1). $\beta$-glycosidases (EC 3.2.1.21) from family 1 of the glycoside hydrolases (GH1) catalyze the hydrolysis of $\beta$-glycosidic bonds, releasing monosaccharides from the non-reducing end of glycosides (5). Their active site is divided into several subsites, which are identified by positive and negative numbers relative to the scissile bond of the substrate (6). Subsite -1 binds the monosaccharide from the non-reducing end of the substrate, also called glycone. Spatial structures of complexes between $\beta$-glycosidases and substrates or inhibitors show that a network of hydrogen bonds is formed between the hydroxyls of the glycone and amino acid residues from subsite -1 (7-11). The energetic contribution of these interactions to the substrate binding has been studied for $\beta$-glucosidase from Agrobacterium $\mathrm{sp}$ (10), lactase-phlorizin hydrolase from lamb (11) and a $\beta$-glycosidase from the fall armyworm Spodoptera fru-

Correspondence: S.R. Marana, Departamento de Bioquímica, Instituto de Química, USP, Caixa Postal 26077, 05513-970

São Paulo, SP, Brasil. Fax: +55-11-3818 2186. E-mail: srmarana@iq.usp.br

*These authors contributed equally to this study.

Received August 18, 2009. Accepted November 27, 2009. Available online December 18, 2009. Published January 11, 2010. 
giperda (Sfßgly) (12).

The energy of interactions of glycone hydroxyls 4 and 6 with residues Q39 (glutamine 39) and E451 (glutamate 451 ) in the ES $\ddagger$ was evaluated in Sf $\beta$ gly (12) and it was shown that these residues are essential elements in determining subsite -1 specificity for fucosides, glucosides, and galactosides. Single mutations of these residues showed that replacement of Q39 with E and N (glutamate and asparagines) causes a drastic decrease (about $70 \%$ ) in the energy of the interactions with the glycone hydroxyls 4 and 6 . In addition, substitution of E451 with $Q$ did not affect the interactions with glycone hydroxyl 4, whereas substitutions with $\mathrm{D}$ and $\mathrm{S}$ (aspartate and serine) produced large reductions in the energy of these interactions (13). However, these interactions were analyzed on a single site mutant so that the effect of mutation of one residue on the interactions formed by a second was not studied. Indeed, the manner in which multiple mutations in the active site are combined and affect the substrate specificity has not been characterized for any $\beta$-glycosidase.

In the present study, in order to identify the influence of interactions formed with the substrate glycone by residues 39 and 451, three double mutants of Sfßgly (A451E39, S451E39 and S451N39) were produced and characterized using enzyme kinetic parameters $\left(k_{\text {cat }} / K_{\mathrm{m}}\right)$ and $\Delta \Delta G^{\ddagger}$ was compared to single mutant (A451, S451, E39, and N39) and wild-type Sfßgly.

\section{Material and Methods}

All reagents, unless otherwise specified, were purchased from Sigma (USA) and Merck (Germany).

\section{Site-directed mutagenesis}

A pT7-7 vector coding the wild-type Sfßgly was used as a template in site-directed mutagenesis experiments employing the kit "QuikChange site directed mutagenesis" (Stratagene, USA). The primer used for mutation at position E451 was 5' GGAGTCTAATGGACAACTTTNNNTGG ATGGAGGGTTATATTGAGCG 3' with TCA and GCC as mutagenic codons (NNN) for S451 and A451, respectively. The primer used for mutation at position Q39 was 5' CGCT ACAGCCTCCTACNNNATCGAAGGTGCTTGG 3' with GAG and ACC as mutagenic codons for E39 and N39, respectively. Thus, to produce a double mutant two different pairs of primers were used in two sequential experiments. The DNA segment coding the double mutant Sfßgly was then amplified by the polymerase chain reaction and cloned in the pET46 vector (Novagen, USA) using the "Ek/LIC cloning" kit (Stratagene). The incorporation of the mutations was confirmed by DNA sequencing.

\section{Production and purification of the Sf $\beta$ gly recombinant \\ BL21 DE3 cells transformed with $p E T 46$ vectors coding}

the double mutant Sfßgly were grown in Luria-Bertani broth containing $50 \mu \mathrm{g} / \mathrm{mL}$ carbenicillin at $37^{\circ} \mathrm{C}$ and $150 \mathrm{rpm}$ until $A_{600}=0.6-1.0$ was obtained. The expression of the Sfßgly recombinant was then induced using $1 \mathrm{mM}$ isopropyl thio- $\beta$ D-galactoside for $3 \mathrm{~h}$ at $25^{\circ} \mathrm{C}$ and $150 \mathrm{rpm}$. The cells were harvested at $7000 \mathrm{~g}$ for $20 \mathrm{~min}$ at $4^{\circ} \mathrm{C}$ and the identity of the recombinant protein was checked by SDS-PAGE (14). The induced bacteria were resuspended in $30 \mathrm{~mL} 20 \mathrm{mM}$ sodium phosphate, $\mathrm{pH} 7.4$, containing $0.5 \mathrm{M} \mathrm{NaCl}, 20 \mathrm{mM}$ imidazole, $0.1 \%$ Triton X-100 (v/v) and $0.2 \%$ chicken hen egg white lysozyme (w/v). After incubation for 30 min with slow shaking (10 rpm), cells were disrupted by sonication (5 pulses of $30 \mathrm{~s}$ at output 4 in a Branson sonifier adapted with a microtip) and the suspension was centrifuged at 7000 $g$ for $20 \mathrm{~min}$ at $4^{\circ} \mathrm{C}$. The supernatant was collected and the Sfßgly recombinant was purified by affinity chromatography on a HisTrap FF column (GE Healthcare, UK). About 15 $\mathrm{mL}$ of the supernatant was passed through $0.22-\mu \mathrm{m}$ filters (Millex, Millipore, USA) and injected into the column. It was eluted with $20 \mathrm{mM}$ sodium phosphate, $\mathrm{pH} 7.4$, containing $0.5 \mathrm{M} \mathrm{NaCl}$ and $20 \mathrm{mM}$ imidazole (flow rate: $1 \mathrm{~mL} / \mathrm{min}$ ). The non-retained proteins were washed out with 15 column volumes of the same buffer. The retained proteins were then eluted with 15 column volumes of $20 \mathrm{mM}$ sodium phosphate, $\mathrm{pH} 7.4$, containing $0.5 \mathrm{M} \mathrm{NaCl}$ and $0.5 \mathrm{M}$ imidazole. Sfßgly activity was detected using $7.2 \mathrm{mM} \mathrm{NP \beta fuc} \mathrm{(p-nitrophenyl}$ $\beta$-D-fucopyranoside; Sigma) prepared in $100 \mathrm{mM}$ citrate phosphate, $\mathrm{pH}$ 6.0. The purification of the Sfßgly mutant was confirmed by SDS-PAGE (14).

Protein was determined on the basis of absorbance at $280 \mathrm{~nm}$. The $\varepsilon_{280}$ of each Sfßgly double mutant was calculated according to Gill and von Hippel (15).

\section{Enzyme kinetic and thermodynamic parameters}

Steady-state kinetic parameters, $k_{\text {cat }}$ and $K_{\mathrm{m}}$, were determined at $30^{\circ} \mathrm{C}$ using at least 10 different substrate concentrations [0.2 to $20 \mathrm{mM}$ for NPßfuc and 0.02 to 8 $\mathrm{mM}$ for NPßgal ( $p$-nitrophenyl $\beta$-D-galactopyranoside)]. Two independent experiments were performed. NPßgal and NPBfuc were prepared in $100 \mathrm{mM}$ citrate-phosphate, $\mathrm{pH}$ 6.0. Substrate hydrolysis was detected by the release of $p$-nitrophenolate by measuring absorbance at $420 \mathrm{~nm}$. Initial rate data were fitted to the Michaelis-Menten equation using the Enzfitter software (R.J. Leatherbarrow; ElsevierBiosoft, UK).

Changes in the free energy $\left(\Delta \Delta G^{\ddagger}\right)$ of the ES $\ddagger$ caused by single or double mutations were calculated using Equation 1 (16):

$\Delta \Delta G^{\ddagger}=-R T \ln \left(k_{\text {cat }} / K_{\mathrm{m} \text { mutant }}\right) /\left(k_{\text {cat }} / K_{\mathrm{m}}\right.$ wild-type $)$

where $R$ is the gas constant, $T$ is the absolute temperature $(303 \mathrm{~K})$, and $k_{\text {cat }} / K_{m}$ is the apparent second-order rate constant of hydrolysis of a substrate. $\Delta \Delta G^{\ddagger}$ represents the difference in free energy between the ES $\$$ complex and the 
free enzyme and substrate $(E+S)$.

The $\Delta \Delta G^{\ddagger}$ caused by a double mutation $\left(\Delta \Delta G^{\ddagger}{ }_{x y}\right)$ can be related to the $\Delta \Delta G^{\ddagger}$ caused by the corresponding single mutations $\left(\Delta \Delta G^{\ddagger}\right.$ and $\Delta \Delta G^{\ddagger}$ ) using Equation 2 (1):

$\Delta \Delta G^{\ddagger} x y=\Delta \Delta G^{\ddagger}+\Delta \Delta G^{\ddagger}+\Delta G^{\ddagger}$

$\Delta G^{\ddagger}$, also known as coupling energy, corresponds to the extent to which the interaction between residues $X$ and $Y$ affects the stabilization of ES $\$$ and consequently the rate of substrate hydrolysis.

\section{Thermal inactivation}

Enzyme samples (wild-type Sfßgly, A451E39 and S451E39) were incubated at $50^{\circ} \mathrm{C}$ for different times ( 0 to $4 \mathrm{~min})$. Next, the remaining activity was determined using $8 \mathrm{mM}$ NPßfuc prepared in $100 \mathrm{mM}$ citrate-phosphate, $\mathrm{pH}$ 6.0. Inactivation rates were compared by plotting the log of the relative remaining activity versus incubation time.

\section{Results and Discussion}

Mutants E39, N39, A451 and S451 (replacement of Sfßgly residues Q39 or E451 by E, N, A, and S, respectively) were previously produced and purified. These single mutations caused a large reduction in the $k_{\text {cat }} / K_{\mathrm{m}}$ ratio for the hydrolysis of NPßglycosides due to a reduction of about $70 \%$ in the energy of the interactions formed by Q39 and E451 with the substrate in the ES $\ddagger$ complex (13).

Double mutants of Sfßgly (A451E39, S451E39 and S451N39) were prepared by site-directed mutagenesis, expressed in BL21 DE3 cells in order to determine interactions between sites of mutation.

Steady-state kinetic parameters $\left(k_{\text {cat }}\right.$ and $\left.K_{\mathrm{m}}\right)$ were determined for the hydrolysis of NPßgal and NPßfuc using a purified Sfßgly double mutant (Table 1). The double mutations modify $k_{\text {cat }} 10^{2}$ - to $10^{3}$-fold, whereas $K_{m}$ presents smaller changes. This pattern was also observed for the single mutations of residues Q39 and E451 $(12,13)$. Thermal inactivation experiments showed that the structures of the A451E39 and S451E39 double mutants are similar to the wild-type Sfßgly $\left(\mathrm{t}_{1 / 2} \sim 1.2 \mathrm{~min}\right.$ at $\left.50^{\circ} \mathrm{C}\right)$, suggesting that the mutational effects on $k_{\text {cat }}$ did not result from large structural modifications of the protein.

On the basis of $k_{\text {cat }} / K_{m}$ data of the wild-type and double mutant Sfßgly, changes in the transition state stabilization energy caused by a double mutation $\left(\Delta \Delta G^{\ddagger_{x y}}\right)$ and by single mutations that compose a double mutant $\left(\Delta \Delta G^{\ddagger_{x}}\right.$ and $\Delta \Delta G^{\ddagger}$ ) were calculated as described in Material and Methods $(1,16)$ (Table 2). These $\Delta \Delta G \ddagger_{x y}$ were compared to the corresponding $\Delta \Delta G^{\ddagger}{ }_{x}$ and $\Delta \Delta G^{\ddagger}$ resulting in the coupling energy $\left(\Delta G \ddagger_{I}\right)$ between residues at sites 451 and 39 of Sfßgly (Table 2 ).

$\Delta G \ddagger_{1}$ is different from zero for all double mutants, except for S451N39 when NPBfuc is used as substrate. Thus, the replacement of E451 and Q39 with S and N, respectively, has a simple additive effect on Sfßgly activity when NPßfuc is the substrate, whereas in all remaining double mutants a complex additive effect is observed. Complex additivity of mutational effects resulting in $\Delta G \ddagger_{\text {I }}$ different from zero has been observed for other enzymes (1-4), but it has not been described for $\mathrm{GH} 1 \beta$-glycosidases.

Table 1. Steady-state kinetic parameters for the hydrolysis of NPßglycosides by the wild-type and double mutant Sfßgly.

\begin{tabular}{lllllllll}
\hline & \multicolumn{4}{c}{$\mathrm{NP \beta gal}$} & & \multicolumn{3}{c}{$\mathrm{NP \beta fuc}$} \\
\cline { 2 - 3 } & $k_{\text {cat }}\left(\mathrm{s}^{-1}\right)$ & $K_{\mathrm{m}}(\mathrm{mM})$ & $k_{\text {cat }} / K_{\mathrm{m}}\left(\mathrm{s}^{-1} \cdot \mathrm{mM}^{-1}\right)$ & & $k_{\text {cat }}\left(\mathrm{s}^{-1}\right)$ & $K_{\mathrm{m}}(\mathrm{mM})$ & $k_{\text {cat }} / K_{\mathrm{m}}\left(\mathrm{s}^{-1} \cdot \mathrm{mM}^{-1}\right)$ \\
\hline Wild type & 0.35 & 2.0 & 0.17 & 3.3 & 0.49 & 6.7 \\
A451E39 & 0.0036 & 0.22 & 0.016 & & 0.0236 & 2.7 & 0.0087 \\
S451E39 & 0.0065 & 0.38 & 0.017 & & 0.0342 & 2.3 & 0.014 \\
S451N39 & 0.0006 & 0.11 & 0.0050 & & 0.00040 & 2.2 & 0.00018 \\
A451 & 0.000285 & 0.50 & 0.00057 & & 0.00061 & 1.53 & 0.0039 \\
E39 & 0.0377 & 2.8 & 0.0134 & & 1.49 & 2.3 & 0.64 \\
S451 & 0.00093 & 1.7 & 0.00053 & & 0.0186 & 0.68 & 0.027 \\
N39 & 0.00038 & 1.5 & 0.00025 & & 0.0220 & 0.26 & 0.084 \\
\hline
\end{tabular}

Experiments were carried out using at least 10 different substrate concentrations. Two independent experiments were performed. Parameters were calculated using the Enzfitter software. Standard errors were less than $12 \%$ of the mean values. Data for wild-type and single mutant A451 were taken from Ref. 12. Data for single mutants E39, S451 and N39 were taken from Ref. 13. $N P \beta g a l=p$-nitrophenyl $\beta$-galactoside $; N P \beta f u c=p$-nitrophenyl $\beta$-fucoside; $A=$ alanine $; E=$ glutamate; $\mathrm{N}=$ asparagine; $\mathrm{S}=$ serine. 
Table 2. Changes in the ES ${ }^{\ddagger}$ complex energy resulting from double and single mutations in Sfßgly.

\begin{tabular}{|c|c|c|c|c|c|c|c|c|}
\hline & \multicolumn{4}{|c|}{ NPßgal } & \multicolumn{4}{|c|}{ NPßfuc } \\
\hline & $\Delta \Delta G_{x y}^{\ddagger_{x y}}(a)$ & $\Delta \Delta G^{ \pm_{X}}(\mathrm{~b})$ & $\Delta \Delta G_{y} \ddagger_{y}(\mathrm{c})$ & $\Delta G^{\ddagger}(\mathrm{d})$ & $\Delta \Delta G^{\ddagger_{x y}}(\mathrm{a})$ & $\Delta \Delta G^{ \pm}{ }_{x}^{(b)}$ & $\Delta \Delta G_{y}^{\ddagger}(\mathrm{c})$ & $\Delta G^{\ddagger}(\mathrm{d})$ \\
\hline A451E39 & 6 & 14 & 6.4 & -14 & 16 & 24 & 6 & -14 \\
\hline S451E39 & 6 & 14 & 6.4 & -14 & 15 & 14 & 6 & -5 \\
\hline S451N39 & 9 & 14 & 16 & -21 & 26 & 14 & 11 & 1 \\
\hline
\end{tabular}

Data are reported as $\mathrm{kJ} / \mathrm{mol}$. ${ }^{a} \Delta \Delta G^{\ddagger}{ }_{x y}$ was calculated by comparing the double mutant and wild-type Sfßgly. ${ }^{b} \Delta \Delta G^{\ddagger} x$ was calculated by comparing a single mutant at position 451 and the wild-type Sf $\beta$ gly. Data from Ref. 13. ${ }^{c} \Delta \Delta G^{\ddagger}$ y was calculated by comparing a single mutant at position 39 and the wildtype Sfßgly. Data from Ref. 13. ${ }^{d} \Delta G \ddagger^{\ddagger}$ was calculated using the equation $\Delta \Delta G^{\ddagger} \ddagger_{x y}=\Delta \Delta G \ddagger_{x}+\Delta \Delta G^{\ddagger} y$ $+\Delta G \ddagger_{I}$ (Ref. 1) and corresponds to the coupling energy between residues at positions 451 and 39 . $\mathrm{NP} \beta g$ al $=p$-nitrophenyl $\beta$-galactoside $; \mathrm{NP} \beta f u c=p$-nitrophenyl $\beta$-fucoside $; \mathrm{A}=$ alanine $; \mathrm{E}=$ glutamate $;$ $\mathrm{N}=$ asparagine; $\mathrm{S}=$ serine .

Negative $\Delta G^{\ddagger}$ indicates that double mutations are less damaging to the enzyme activity (measured by $k_{\text {cat }} / K_{\mathrm{m}}$ ) than the sum of the single mutations that compose them $\left(\Delta \Delta G^{\ddagger} x y=\Delta \Delta G^{\ddagger} x+\Delta \Delta G^{\ddagger} y\right)$. This difference also indicates that the effects of the single mutations partially overlap. Hence, this common effect counts just once in the $\Delta \Delta G^{\ddagger} x y$. Moreover, this common effect could result from the presence of a mutual influence between residues at positions 451 and 39, which affects their bonds with the substrate and favors substrate hydrolysis.

In the specific case of NPßgal hydrolysis, $\Delta G^{\ddagger}$, presents similar values for all double mutants. In addition, the effects of the double mutations $\left(\Delta \Delta G^{\ddagger} x y\right)$ are less than those of the most damaging single mutations $\left(\Delta \Delta G{ }_{x}\right)$, which involve residue 451 (Table 2). Thus, mutations at residue 39 are partially suppressing the damaging effect of the mutations at residue 451 , which is compatible with a mutual influence between residues at positions 451 and 39 .

On the other hand, in the case of NPßfuc hydrolysis, $\Delta G^{\ddagger}$ l changes depending on the double mutations (Table 2). Variation of $\Delta G^{\ddagger}$ l depending on the type of residue introduced in the mutant enzyme was also observed for tyrosyl-tRNA synthetase and subtilisin BPN' mutants (1). In the A451E39 mutant the observation of a negative $\Delta G^{\ddagger}$ I and a $\Delta \Delta G^{\ddagger}$ xy lower than $\Delta \Delta G^{\ddagger}{ }_{x}$ suggests that the introduction of E39 is reducing the damage caused by mutation A451, an effect similar to that described for the hydrolysis of NPßgal by this same double mutant. The suppressing effect completely disappears in the mutants S451E39 and S451N39 when NPßfuc is the substrate. In spite of that, the $\Delta \Delta G{ }_{x y}$ is still lower than $\Delta \Delta G^{\ddagger} x+\Delta \Delta G^{\ddagger}$ for mutants S451E39 and S451N39, confirming the presence of a mutual influence between residues at positions 451 and 39.

Therefore, the present data indicate a mutual effect of residues occupying positions 39 and 451 on their interactions with the substrate. According to previous observations, negative $\Delta G \ddagger_{\text {I }}$ occurs when the mutated residues form direct or indirect interactions (1). Crystallographic data for $\beta$-glycosidases show that residues corresponding to Q39 and E451 do not interact directly because they are more than $3 \AA$ apart. Nevertheless these residues form bidentate hydrogen bonds with the hydroxyl 4 of the glycone substrate (17-20). As the hydroxyl 4 is simultaneously bound to Q39 and E451, these interactions are not isolated. Thus, interacting with the same group within the substrate rather than with different groups may result in different interaction energies. Hence, the sharing of the same group within the substrate may be the source of the mutual influence between residues Q39 and E451 for Sfßgly.

Comparison of sequence data shows that residues forming the subsite -1 are highly conserved among the GH1 $\beta$-glycosidases (17) and several of them share a common group (glycone hydroxyl) in their interactions with the substrate $(9,17-20)$, suggesting that a mutual influence between residues involved in substrate binding may be a common characteristic within this group of enzymes. Therefore, this property should be considered in the characterization and/or modification of the substrate specificity of GH1 $\beta$-glycosidases.

\section{Acknowledgments}

Research supported by FAPESP and CNPq. M.H. Tomassi is the recipient of an undergraduate fellowship from CNPq. J.H.K. Rozenfeld and L.M. Gonçalves are recipients of graduate fellowships from CNPq. S.R. Marana is a CNPq research fellow. 


\section{References}

1. Wells JA. Additivity of mutational effects in proteins. Biochemistry 1990; 29: 8509-8517.

2. Kuliopulos A, Talalay P, Mildvan AS. Combined effects of two mutations of catalytic residues on the ketosteroid isomerase reaction. Biochemistry 1990; 29: 10271-10280.

3. Mildvan AS, Weber DJ, Kuliopulos A. Quantitative interpretations of double mutations of enzymes. Arch Biochem Biophys 1992; 294: 327-340.

4. Mildvan AS. Inverse thinking about double mutants of enzymes. Biochemistry 2004; 43: 14517-14520.

5. Coutinho P, Henrissat B. Carbohydrate-active enzymes: an integrated database approach. In: Gilbert HJ, Davies G, Henrissat B, Svensson B (Editors), Recent advances in carbohydrate bioengineering. Cambridge: The Royal Society of Chemistry; 1999. p 3-12.

6. Davies GJ, Wilson KS, Henrissat B. Nomenclature for sugarbinding subsites in glycosyl hydrolases. Biochem $J$ 1997; 321 (Part 2): 557-559.

7. Burmeister WP, Cottaz S, Driguez H, lori R, Palmieri S, Henrissat B. The crystal structures of Sinapis alba myrosinase and a covalent glycosyl-enzyme intermediate provide insights into the substrate recognition and active-site machinery of an S-glycosidase. Structure 1997; 5: 663-675.

8. Cicek M, Blanchard D, Bevan DR, Esen A. The aglycone specificity-determining sites are different in 2, 4-dihydroxy7-methoxy-1,4-benzoxazin-3-one (DIMBOA)-glucosidase (Maize beta-glucosidase) and dhurrinase (Sorghum betaglucosidase). J Biol Chem 2000; 275: 20002-20011.

9. Gloster TM, Roberts S, Ducros VM, Perugino G, Rossi M, Hoos R, et al. Structural studies of the beta-glycosidase from Sulfolobus solfataricus in complex with covalently and noncovalently bound inhibitors. Biochemistry 2004; 43: 6101-6109.

10. Fernandez P, Canada FJ, Jimenez-Barbero J, Martin-Lomas M. Substrate specificity of small-intestinal lactase: study of the steric effects and hydrogen bonds involved in enzymesubstrate interaction. Carbohydr Res 1995; 271: 31-42.

11. Namchuk MN, Withers SG. Mechanism of Agrobacterium beta-glucosidase: kinetic analysis of the role of noncova- lent enzyme/substrate interactions. Biochemistry 1995; 34: 16194-16202.

12. Marana SR, Terra WR, Ferreira C. The role of amino-acid residues Q39 and E451 in the determination of substrate specificity of the Spodoptera frugiperda beta-glycosidase. Eur J Biochem 2002; 269: 3705-3714.

13. Marana SR, Andrade EH, Ferreira C, Terra WR. Investigation of the substrate specificity of a beta-glycosidase from Spodoptera frugiperda using site-directed mutagenesis and bioenergetics analysis. Eur J Biochem 2004; 271: 41694177.

14. Laemmli UK. Cleavage of structural proteins during the assembly of the head of bacteriophage T4. Nature 1970; 227 : 680-685.

15. Gill SC, von Hippel PH. Calculation of protein extinction coefficients from amino acid sequence data. Anal Biochem 1989; 182: 319-326

16. Wilkinson AJ, Fersht AR, Blow DM, Winter G. Site-directed mutagenesis as a probe of enzyme structure and catalysis: tyrosyl-tRNA synthetase cysteine-35 to glycine-35 mutation. Biochemistry 1983; 22: 3581-3586.

17. Marana SR. Molecular basis of substrate specificity in family 1 glycoside hydrolases. IUBMB Life 2006; 58: 63-73.

18. Sanz-Aparicio J, Hermoso JA, Martinez-Ripoll M, Lequerica $\mathrm{JL}$, Polaina J. Crystal structure of beta-glucosidase A from Bacillus polymyxa: insights into the catalytic activity in family 1 glycosyl hydrolases. J Mol Biol 1998; 275: 491-502.

19. Czjzek M, Cicek M, Zamboni V, Burmeister WP, Bevan DR, Henrissat $B$, et al. Crystal structure of a monocotyledon (maize ZMGlu1) beta-glucosidase and a model of its complex with p-nitrophenyl beta-D-thioglucoside. Biochem $J$ 2001; 354: 37-46.

20. Verdoucq L, Moriniere J, Bevan DR, Esen A, Vasella A, Henrissat $B$, et al. Structural determinants of substrate specificity in family 1 beta-glucosidases: novel insights from the crystal structure of sorghum dhurrinase-1, a plant beta-glucosidase with strict specificity, in complex with its natural substrate. $J$ Biol Chem 2004; 279: 31796-31803. 